\title{
Produção científica sobre profissional biomédico e promoção da saúde: uma revisão
}

\section{de escopo}

\author{
Scientific production about biomedical professionals and health promotion: a scoping review \\ Producción científica sobre profesionales biomédicos y promoción de la salud: revisión exploratoria
}

Recebido: 05/02/2022 | Revisado: 15/02/2022 | Aceito: 21/02/2022 | Publicado: 03/03/2022

\author{
Vanessa Gomes da Costa \\ ORCID: https://orcid.org/0000-0002-1123-8597 \\ Universidade Federal do Estado do Rio de Janeiro, Brasil \\ E-mail: vanessagcosta@edu.unirio.br \\ Mariana Soares da Silva Peixoto Belo \\ ORCID: https://orcid.org/0000-0001-9666-284X \\ Universidade Federal do Estado do Rio de Janeiro, Brasil \\ E-mail: mariana.belo@unirio.br \\ Tatiana Pereira das Neves Gamarra \\ ORCID: https://orcid.org/ 0000-0002-2773-5133 \\ Agência Nacional de Saúde Suplementar, Brasil \\ E-mail: tatibiom@yahoo.com.br
}

\begin{abstract}
Resumo
Como profissional da saúde, o biomédico deve ter conhecimento acerca do conceito e das estratégias da promoção da saúde para atuarem com tal orientação, por meio do exercício das práticas e formas de diálogo com a sociedade, aprimorando essas estratégias de acordo com o contexto atual. Nesse sentido, o estudo tem como objetivo analisar o que a literatura apresenta sobre a relação entre o profissional biomédico e a promoção da saúde. Trata-se de revisão de escopo que teve a seguinte pergunta norteadora: Como se caracteriza a produção científica brasileira sobre profissional biomédico e promoção da saúde em revistas nacionais a partir do ano de 1999? Mesmo que uma parcela dos artigos encontrados tenha foco na atuação do biomédico, como identificado na análise, todos indicam fragilidades e efeitos oriundos da formação acadêmica. Desse modo, foi indicado que uma maior integração entre os cursos da área da saúde, vivência prática com a aplicação para a sociedade, estudos com perspectiva antropológica e ênfase na interdisciplinaridade auxiliariam em uma formação mais ampliada para o exercício do profissional biomédico na promoção da saúde.
\end{abstract}

Palavras-chave: Profissional biomédico; Promoção da saúde; Revisão de escopo.

\begin{abstract}
As a health professional, biomedical practitioners must have knowledge about the concept and strategies of health promotion to act with such guidance, through the exercise of practices and forms of dialogue with society, improving these strategies according to the current context. In this sense, the study aims to analyze what the literature presents about the relationship between the biomedical professional and health promotion. This is a scope review that had the following guiding question: How is the Brazilian scientific production on biomedical professionals and health promotion characterized in national journals since 1999? Even though a portion of the articles found focus on the role of the biomedic, as identified in the analysis, all indicate weaknesses and effects arising from academic training. Thus, it was indicated that greater integration between courses in the health area, practical experience with application to society, studies with an anthropological perspective and an emphasis on interdisciplinarity would help in a broader training for the exercise of the biomedical professional in health promotion.
\end{abstract}

Keywords: Biomedical professional; Health promotion; Scoping review.

\section{Resumen}

Como profesional de la salud, los biomédicos deben tener conocimientos sobre el concepto y las estrategias de la promoción de la salud para actuar con dicha orientación, a través del ejercicio de prácticas y formas de diálogo con la sociedad, perfeccionando estas estrategias de acuerdo al contexto actual. En este sentido, el estudio tiene como objetivo analizar lo que presenta la literatura sobre la relación entre el profesional biomédico y la promoción de la salud. Se trata de una revisión exploratoria que tuvo la siguiente pregunta orientadora: ¿Cómo se caracteriza la producción científica brasileña sobre profesionales biomédicos y promoción de la salud en las revistas nacionales desde 1999? Si bien una parte de los artículos encontrados se centran en el papel de la biomedicina, tal como se identifica en el análisis, todos señalan debilidades y efectos derivados de la formación académica. Así, se indicó que una mayor integración entre cursos en el área de la salud, experiencia práctica con aplicación a la sociedad, estudios 
con perspectiva antropológica y un énfasis en la interdisciplinariedad ayudaría en una formación más amplia para el ejercicio del profesional biomédico en la promoción de la salud.

Palabras clave: Profesional biomédico; Promoción de la salud; Revisión exploratoria.

\section{Introdução}

As Diretrizes Curriculares Nacionais do Cursos de Graduação em Biomedicina preconizam um ensino interdisciplinar em consonância com as expectativas da formação de um profissional da saúde capaz de trabalhar no Sistema Único de Saúde (SUS). Portanto, contempla a abrangência do cuidado exposto na lei 8.080 e considera o conceito amplo de saúde. Desse modo, faz-se necessário discutir a presença de um importante tema durante a graduação: a promoção da saúde. Desde a Carta de Ottawa de 1986, documento redigido a partir da Primeira Conferência Internacional sobre Promoção da Saúde, aconteceram diversos encontros, que contribuíram, posteriormente, para o debate sobre a promoção da saúde propondo práticas para promover qualidade de vida e equidade em saúde (Heidmann et al., 2006). O contexto histórico-legal é importante para compreender as modificações do curso. Assim, vale destacar que, em 1998, o biomédico foi reconhecido como profissional da saúde, em 2003 foram publicadas as Diretrizes Curriculares; e em 2010, a $3^{\text {a }}$ edição da Política Nacional de Promoção da Saúde.

A promoção da saúde abrange aspectos econômicos, sociais, culturais, políticos e ambientais e, de acordo com o conteúdo apresentado nas Diretrizes Curriculares do curso, o biomédico pode ser capaz de exercer sua profissão considerando esses fatores, já que a Biomedicina abarca necessariamente a interdisciplinaridade.

Além disso, dentro do conceito de promoção da saúde e educação da saúde, é fundamental que o estudante tenha contato com campos que levem em conta o conceito ampliado de saúde; a intersetorialidade; a educação em saúde e o empowerment; a base social e coletiva. A prática da promoção da saúde é fundamentada em cinco eixos: elaboração e implementação de políticas públicas saudáveis; criação de ambientes favoráveis à saúde; reforço da ação comunitária; desenvolvimento de habilidades pessoais; e reorientação do sistema de saúde (Buss, 2000).

Como profissional da saúde, o biomédico deve ter conhecimento acerca do conceito e das estratégias para serem promotores de saúde, por meio do exercício das práticas e formas de diálogo com a sociedade, para aprimorar tais estratégias de acordo com o contexto atual. Ademais, o desconhecimento dos conceitos de saúde e promoção da saúde entre os profissionais (Heidmann et al., 2006), revela a importância de a formação dos estudantes estar de acordo com as propostas para o SUS. Contudo, tanto as Diretrizes Curriculares quanto a Política Nacional de Promoção da Saúde não explicitam, de forma objetiva, competências e habilidades que um profissional deve desenvolver, gerando possíveis impactos negativos para a formação dos estudantes da área da saúde (Pinheiro et al., 2015).

É possível citar que ligas acadêmicas, projetos de extensão e outras atividades complementares possuem um papel fundamental para colaborar com a formação do estudante, já que exercem de forma mais aplicada uma ligação prática entre os estudantes e a sociedade, ampliando o conhecimento sobre diversos temas em um formato menos conteudista. Para o desenvolvimento de competências para a promoção da saúde é primordial a imersão do aluno em práticas que envolvam o conteúdo teórico de maneira contextualizada. Todavia, é visto que a graduação não prepara totalmente para o exercício da profissão como promotora da saúde, já que os cursos ainda apresentam conteúdo sem uma abordagem com real contextualização das necessidades da sociedade e seguem com foco no modelo biomédico (Chiesa et al, 2007).

Pinheiro et al. (2015) discutem que no Brasil ainda há poucos estudos na literatura sobre competências dos profissionais para serem promotores de saúde, que além de estabelecer padrões para exercer a profissão e garantir a qualidade dos serviços oferecidos, também possuam como função orientar a formação desses profissionais.

A aplicação de práticas promotoras da saúde é dificultada pela maior existência de ações que consideram a saúde como ausência de doença de modo restrito e muitas vezes as ações são baseadas nessa compreensão limitada (Bezerra \& 
Sorpreso, 2016). Tal fato pode evidenciar uma falha na formação dos profissionais da saúde.

O empowerment psicológico e social são conceitos em que o indivíduo e a comunidade desenvolvem autonomia para agir no seu próprio processo de saúde (Souza et al., 2014). Essa prática traz um eixo de atuação para o profissional biomédico junto à educação emancipadora que propiciam poder ao sujeito para realizar ações que busquem qualidade de vida (Tesser, 2009).

Nesse sentido, essa revisão tem como objetivo analisar o que a literatura apresenta sobre a relação entre o profissional biomédico e a promoção da saúde no contexto da graduação, o que os estudos sobre o tema trazem de proximidade do estudante com o conceito de promoção da saúde e como ele é abordado durante a graduação. Nessa perspectiva, serão discutidos os estudos encontrados sobre o tema na Biomedicina e destacados os pontos de convergência e divergência na formação do profissional capaz de atuar como promotor da saúde.

\section{Metodologia}

A revisão de escopo foi escolhida a fim de responder ao questionamento principal proposto que consiste em analisar a aproximação da temática com o curso e identificar destaques que seriam essenciais para a formação do biomédico.

Uma revisão de escopo deve ser realizada quando a pesquisa possuir alguns dos seguintes propósitos (Munn et al., 2018):

- Identificar os tipos de evidências disponíveis em um determinado campo.

- $\quad$ Esclarecer os principais conceitos/definições na literatura.

- Examinar como a pesquisa é conduzida em um determinado tópico ou campo.

- Identificar as principais características ou fatores relacionados a um conceito.

- $\quad$ Funcionar como um precursor para uma revisão sistemática.

- Identificar e analisar lacunas de conhecimento.

O presente estudo segue as orientações Preferred Reporting Items for Systematic Reviews and Meta-Analyses PRISMA ScR para revisão de escopo (Tricco et al., 2018). Essa extensão possui uma lista de 22 itens a serem cumpridos pela revisão, abrangendo todas as etapas da redação. Dessa forma, primeiramente foram definidos: população, conceito, contexto e os critérios para a análise da bibliografia. Foi delimitada a população: profissionais de biomedicina, o conceito estudado: promoção da saúde e o contexto: Brasil.

Dessa forma, será possível responder ao amplo questionamento proposto mapeando os pontos anteriormente indicados de forma objetiva e, portanto, sistematizar as evidências conhecidas e identificar as lacunas presentes. A seguinte pergunta norteadora foi definida: "Como se caracteriza a produção científica brasileira sobre o profissional biomédico e a promoção da saúde em revistas nacionais a partir do ano de 1999?"

As Diretrizes Curriculares da Biomedicina (Resolução $n^{\circ}$ 2, de 18 de fevereiro de 2003) foram utilizadas como referencial teórico para destacar as especificidades desse curso. No conceito estudado foram analisados os eixos da saúde e educação que envolvem a aplicação da promoção da saúde perante às definições teóricas de saúde. Diante das bases conceituais, foram expostas as utilizadas pela Política Nacional de Promoção da Saúde e destacado a inclusão desses pontos nas Diretrizes Curriculares, visto que o contexto busca evidenciar a presença do conceito promoção da saúde durante a graduação.

A busca foi realizada nas seguintes plataformas: Scientific Electronic Library Online - SciELO, PubMed/Medical Literature Analysis and Retrieval System Online - MEDLINE,Literatura Latino-americana e do Caribe em Ciências da Saúde - 
LILACS, , Directory of Open Access Journals - DOAJ, Red Iberoamericana de Innovación y Conocimiento Científico REDIB, Scientific Literature - Scilit, Sumários, Red de Revistas Científicas de América Latina y el Caribe, España y Portugal - Redalyc, Servicio de Difusión de Alertas en la Red - Dialnet, Periódicos CAPES e Google Acadêmico.

O Google Acadêmico foi utilizado porque, segundo Larson et al. (2019), possui cobertura mais abrangente que as bases de dados convencionais, podendo encontrar um maior número de artigos. Os artigos selecionados foram publicados a partir do ano de 1999 para estar no critério estabelecido, isto é, após a data na qual o biomédico foi considerado profissional da saúde, 8 de outubro de 1998, por meio da publicação da Resolução n²87. Dessa maneira, os estudos analisados são posteriores ao grande marco na regulamentação da profissão. A busca foi realizada por meio das palavras-chave: "profissional biomédico" "promoção da saúde" de forma simultânea em todos os campos de busca disponíveis nas fontes de dados pesquisadas.

\section{Resultados e Discussão}

A busca foi realizada em 20 de julho de 2021. Cento e cinquenta e uma publicações foram identificadas nas diferentes bases e de dados e diretórios utilizados. Após a seleção, foram identificados 15 artigos que cumprem com os objetivos deste estudo. 
Figura 1 - Fluxograma com as etapas do levantamento bibliográfico: identificação, seleção, elegibilidade e inclusão.

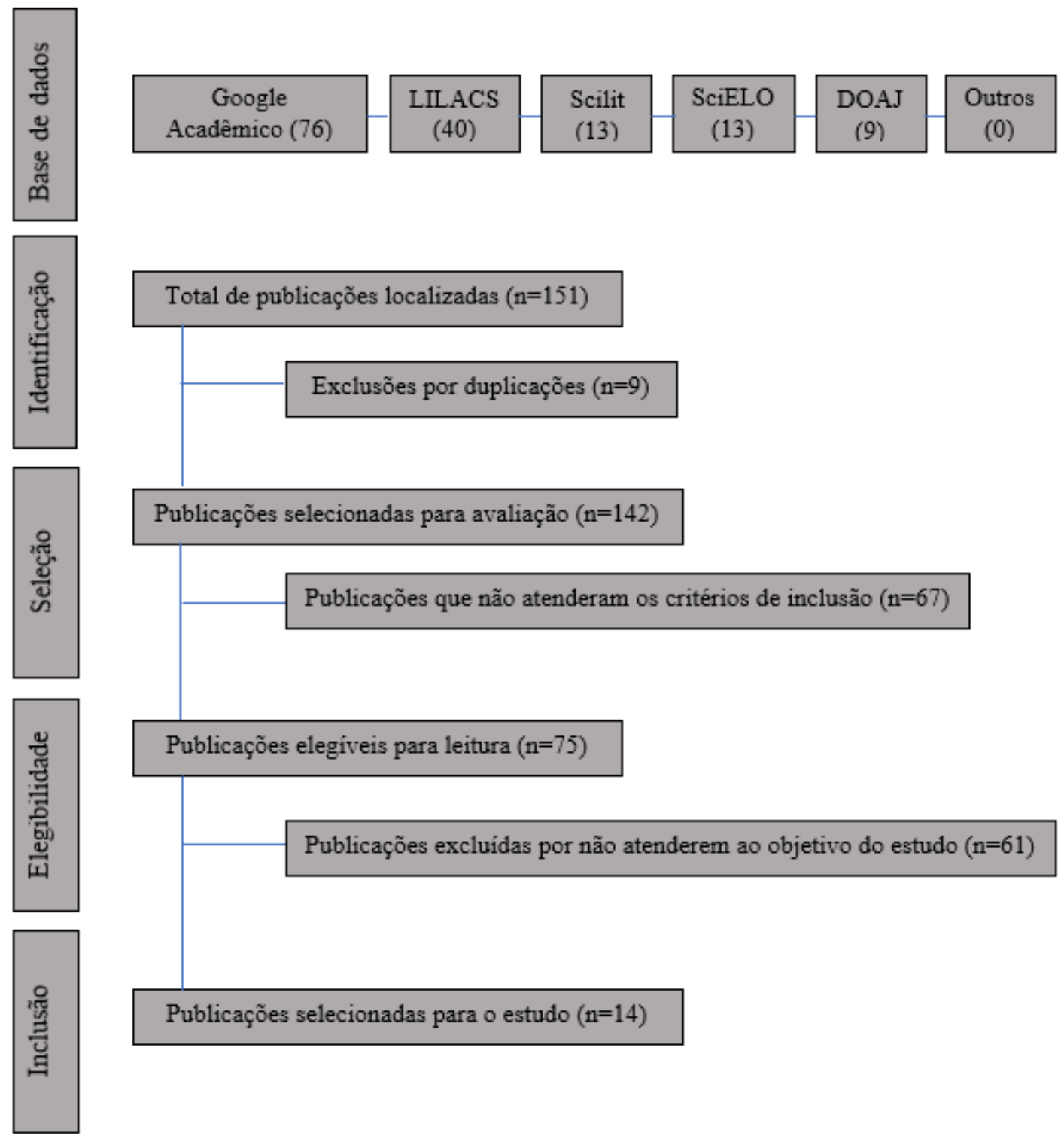

Fonte: Elaboração própria.

Após o levantamento realizado nas bases de dados, foram excluídas as duplicações, ou seja, artigos que aparecem mais de uma vez na mesma ou em plataformas diferentes. Em seguida, foi analisado se os artigos selecionados atendem aos critérios de seleção, dentre eles sessenta e sete foram excluídos por não serem artigos científicos. Os setenta e cinco artigos restantes foram lidos e, então, selecionados para a inclusão no estudo quatorze artigos em português, com data de publicação entre 2004 a 2020 e que discutem promoção da saúde para a formação e atuação do profissional biomédico. O resultado da busca pode ser visualizado na Tabela 1, onde há o título, revista, data das publicações e tema. 
Tabela 1 - Artigos que relacionam o profissional biomédico à promoção da saúde.

\begin{tabular}{|c|c|c|c|c|}
\hline Título & Método & Tema & Revista & Ano \\
\hline $\begin{array}{l}\text { A atuação do profissional biomédico na Atenção Primária à Saúde: } \\
\text { desafios na formação }\end{array}$ & Qualitativo & $\begin{array}{l}\text { Atuação e } \\
\text { formação }\end{array}$ & Revista Saúde Integrada & 2015 \\
\hline A fitoterapia entre acadêmicos das ciências da vida & $\begin{array}{l}\text { Quali- } \\
\text { quantitativo }\end{array}$ & Formação & Revista Saúde e Desenvolvimento & 2017 \\
\hline $\begin{array}{l}\text { A importância do profissional biomédico na prática de Cuidados } \\
\text { Paliativos no tratamento oncológico }\end{array}$ & Qualitativo & Atuação & Brazilian Journal of Development & 2020 \\
\hline A inserção do biomédico no Programa de Saúde da Família & Qualitativo & Atuação & Revista Eletrônica Novo Enfoque & 2010 \\
\hline $\begin{array}{l}\text { A integração da Educação Ambiental na formação do biomédico } \\
\text { em uma Universidade comunitária: indícios a partir dos Princípios } \\
\text { da Ambientalização Curricular }\end{array}$ & Qualitativo & Formação & $\begin{array}{l}\text { REMEA - Revista Eletrônica do } \\
\text { Mestrado em Educação Ambiental }\end{array}$ & 2018 \\
\hline Atenção as ações comunitárias na formação de biomédicos & $\begin{array}{l}\text { Quali- } \\
\text { quantitativo }\end{array}$ & Formação & $\begin{array}{c}\text { Revista Brasileira de Ciências } \\
\text { Biomédicas }\end{array}$ & 2020 \\
\hline $\begin{array}{l}\text { Avaliação do conhecimento de professores universitários de cursos } \\
\text { de biomedicina sobre educação em saúde }\end{array}$ & $\begin{array}{l}\text { Quali- } \\
\text { quantitativo }\end{array}$ & Formação & Temas em Saúde & 2017 \\
\hline $\begin{array}{l}\text { Fatores associados à ocorrência de ansiedade dos acadêmicos de } \\
\text { biomedicina }\end{array}$ & $\begin{array}{l}\text { Quali- } \\
\text { quantitativo }\end{array}$ & Formação & Saúde e Pesquisa & 2016 \\
\hline $\begin{array}{l}\text { Inserção do biomédico nas equipes multiprofissionais da Estratégia } \\
\text { de Saúde da Família e Núcleo de Apoio à Saúde da Família }\end{array}$ & Qualitativo & Atuação & Revista Saúde Integrada & 2019 \\
\hline $\begin{array}{l}\text { Liga acadêmica de plantas medicinais: } \\
\text { um desafio extracurricular }\end{array}$ & Qualitativo & Formação & $\begin{array}{c}\text { Extensio: Revista Eletrônica de } \\
\text { Extensão }\end{array}$ & 2019 \\
\hline O papel do biomédico na análise ambiental & Qualitativo & Atuação & Cadernos de Graduação & 2015 \\
\hline O papel do biomédico na Saúde Pública & Qualitativo & Atuação & Revista Interfaces & 2014 \\
\hline Projeto Cidadania: formação do profissional biomédico & Qualitativo & Formação & Revista Em Extensão & 2004 \\
\hline $\begin{array}{l}\text { Trabalho em equipe multiprofissional na saúde: da concepção ao } \\
\text { desafio do fazer na prática }\end{array}$ & Qualitativo & Formação & Disciplinarum Scientia Saúde & 2014 \\
\hline
\end{tabular}

Fonte: Elaboração própria.

A tabela com os artigos incluídos exibe os métodos dos estudos, sendo qualitativo (10), quantitativo (0) ou qualiquantitativo (4) e os temas abordados que classificam os resultados em categorias: formação acadêmica (8), atuação do profissional (5) ou ambos (1). O intervalo de tempo das publicações foi de 2004 a 2020.

Diante dos resultados, é possível destacar que dois artigos possuem como tema a participação ativa do biomédico no campo ambiental, em que um aborda a presença da disciplina de Educação Ambiental durante a graduação e o outro discute a atuação do profissional na análise ambiental. Ambos corroboram com a relevância da biomedicina para o meio ambiente relacionando com a promoção da saúde da população. No entanto, essa discussão também está presente em outros artigos, Costa et al. (2010) apresenta um exemplo:

"Destacou-se ainda que o trabalho do biomédico não se restringe apenas à identificação dentro de um laboratório dos agentes etiológicos que podem causar doenças na comunidade. Ele também pode e deve atuar como orientador no descarte adequado desses dejetos que podem contaminar o meio ambiente e trazer sérias consequências à saúde das pessoas"

Não apenas esse trecho, mas o artigo intitulado "O papel do biomédico na análise ambiental" também afirma que um dos campos de maior destaque para o biomédico é voltado à qualidade de água e saneamento. Além disso, a atuação do 
biomédico é indicada para proporcionar um equilíbrio entre a sociedade e o meio ambiente para que proporcione a manutenção da qualidade de vida da população e menor impacto ambiental (Silva et al., 2015).

A educação ambiental no currículo do curso de Biomedicina é responsável por uma visão socioambiental do estudante, que irá desenvolver competências que implicam no âmbito individual e coletivo, além de inserir os demais aspectos da saúde (Pessoa, et al, 2018). Além disso, o estudo de Pessoa et al., (2018) faz uma análise dos pontos das Diretrizes Curriculares Nacionais (DCN) que coincidem com os Princípios da Ambientalização Curricular, portanto encontram convergências, mas também lacunas como a ausência da inclusão dos assuntos: diversidade de gênero, religião e culturas e mudanças climáticas.

Ademais, foi encontrado a análise da saúde dos estudantes, como futuros profissionais da saúde, indicando aspectos psicológicos afetados durante uma fase da formação. Dessa forma, é explicitado o cuidado que o estudante deve desenvolver consigo para, também, cuidar do outro, estabelecendo como relevante o conhecimento para proporcionar o desenvolvimento do seu próprio bem estar (Cardozo et al., 2016). Outra publicação selecionada (Perinazzo et al., 2015), também, discute sobre a saúde do estudante, trazendo a importância de lidar com o estresse do profissional durante a graduação.

Dentre os artigos sobre a formação do biomédico, quatro desses discutem a necessidade da extensão e/ou estágio durante a graduação para o exercício da prática contextualizada, aproximando o estudante da realidade da população. Já nos artigos sobre a atuação do profissional, quatro discutem sobre o trabalho em equipes ou especificam a presença do biomédico em equipes multiprofissionais. Outra questão muito enfatizada, é a necessidade da atuação humanizada que dialoga com uma formação mais próxima da realidade durante a graduação, tema relevante, também, para o cuidado equitativo proposto pelo SUS.

De forma objetiva, os artigos citam a atuação do biomédico na epidemiologia, vigilância sanitária, educação em saúde através de palestras, orientações e grupos terapêuticos, assessorias, gestão, Práticas Integrativas e Complementares em Saúde (PICS) e no laboratório clínico. É visto que não ocorre uma definição precisa das práticas promotoras de saúde realizadas pelo profissional biomédico, tornando tênue o exercício entre prevenção e promoção da saúde.

A distinção entre promoção e prevenção é maior no campo teórico do que na prática (Czeresnia, 2003), tendo em vista que, inicialmente, a promoção da saúde é uma medida de prevenção primária (Leavel \& Clark apud Czeresnia, 2003). Todavia, é compreendido que a promoção da saúde envolve campos para além da saúde, de forma mais direta, diferente da prevenção. Ao englobar a definição de saúde indicada pela Política Nacional de Promoção da Saúde, é visto que se faz necessário a participação da política, da economia, do ambiente, das condições trabalhistas e psicossociais. Além disso, o motivo pelo qual a diferença não se apresenta de forma tão evidente é que as práticas em saúde são científicas (Czeresnia, 2003), no entanto, o conceito de saúde envolve também questões não-científicas (Almeida Filho, 2011). Essa complexidade da saúde justifica a necessidade da antropologia e da interdisciplinaridade no currículo dos cursos de biomedicina como citado em alguns dos artigos incluídos, colaborando para o desenvolvimento das competências descritas nas DCN do curso.

No que diz respeito à formação dos estudantes, foi indicado que há o distanciamento entre a teoria aprendida nas salas de aula e a prática, sugerindo a insuficiência de interdisciplinaridade e a associação com o contexto de populações específicas. Para tais questões, a indicação de atividades extensionistas obtiveram resultados positivos para a formação dos profissionais da saúde, como apontam diversos estudos analisados: Rodrigues et al., 2018; Oliveira et al., 2021; Silva et al., 2019

Ademais, referente às ações da promoção da saúde, faz-se necessária uma discussão sobre a importância da educação em saúde para a população. Dessa forma, o biomédico pode atuar como promotor da saúde utilizando o empowerment para dar ferramentas para o indivíduo e para a comunidade construírem independência e análise crítica sobre os determinantes e condicionantes da saúde assumindo controle no desenvolvimento da qualidade de vida (Carvalho, 2004). Além disso, como visto anteriormente, há discussões sobre os aspectos ambientais da saúde. No entanto, não ocorre discussões sobre os aspectos 
políticos e econômicos, no que resguarda a formação e atuação do profissional da saúde, mesmo que citado o exercício do biomédico na gestão.

Perinazzo et al., (2015) destacam a formação voltada para o desenvolvimento de habilidades referentes ao trabalho em equipe, principalmente aspectos éticos, para que a prática não traga prejuízos para a população. Dessa forma, o profissional deve ter as competências necessárias para realizar um cuidado integral e humanizado com a interação de diversos profissionais capazes de atuarem juntos na complexidade das questões relacionadas à saúde humana, afim de proporcionar qualidade de vida para além de tratamento e recuperação dos pacientes. Porém, também enfatiza que a ausência de profissionais suficientes compondo uma equipe para atender uma determinada população é um dos fatores que prejudica a atenção à saúde e implica uma sobrecarga aos que atendem um número elevado de usuários.

Ademais, outro estudo (Backes et al., 2014) aborda os reflexos da formação para o trabalho em equipe multidisciplinar e indica que as DCN explicitam competências e habilidades que retificam o ensino baseado no modelo biomédico e direcionam a formação para a plena atuação no sistema de saúde. Por meio desse estudo, é visto também que a percepção da complexidade da formação proposta pelas DCN não apresenta de forma clara para o desenvolvimento de tais competências e habilidades, também evidenciado na literatura (Pinheiro et al., 2015). Além disso, esse artigo aborda a complexidade da saúde, criticando o aprendizado com foco em uma especialidade e, assim como outros aqui citados, foram destacadas a interdisciplinaridade e a educação permanente como estratégias de formação para um cuidado integral: Costa $e t$ al., 2010; Silva \& Júnior, 2019; Pessoa et al., 2018; Perinazzo et al., 2015; Santos \& Melo, 2020; Silva et al., 2014.

Já o artigo sobre fitoterápicos relaciona classe social com o uso de produtos naturais, bem como, evidencia a ausência de informação sobre o uso adequado. Por serem produtos difundidos popularmente e de fácil acesso, a inclusão nas PICS é um meio de informar e transpor educação em saúde para o uso dessa ferramenta terapêutica. Destaca ainda que a adesão ao uso de fitoterápicos e plantas medicinais é alta, porém falta informação por parte dos profissionais da saúde (Faria et al., 2017).

No mais, Silva et al. (2015), também corroboram com a facilidade de adesão aos fitoterápicos e plantas medicinais. Além dos benefícios, o artigo introduz esse tema de estudo para compor a Liga Acadêmica que insere o graduando na atividade de ensino, pesquisa e extensão. Dessa forma, os alunos podem exercer a prática para a sociedade, estudar o tema com profundidade e compartilhar o espaço com estudantes de outros cursos da área, já que Farmacêuticos, Biomédicos, Nutricionistas, Fisioterapeutas, Odontólogos e Enfermeiros podem atuar com fitoterápicos.

Outro tema abordado em um artigo, cuidados paliativos, inclui aspectos físicos, psicossociais e espirituais que promovem qualidade de vida para pacientes que possuem doenças graves. Assim como nas PICS, é necessário o acompanhamento por uma equipe multiprofissional, dada ao entendimento de diversos fatores condicionantes para o bem-estar do usuário. O biomédico atua nessa área impactando na promoção da saúde do paciente por meio da análise clínica, podendo ter contato direto através da coleta do material biológico, dessa forma, o profissional atua na análise de dados clínicos e no intercâmbio de informações entre a equipe e com o paciente. Santos e Mello (2016) expõem que o biomédico não é citado em nenhum artigo selecionado para o estudo, mesmo podendo integrar as equipes multiprofissionais.

Costa et al. (2020) ressaltam que o biomédico é um profissional novo no mercado de trabalho. Dessa forma, é entendido a inserção gradual da biomedicina e, é possível que o cenário exposto pelas publicações, em que há falta de conhecimento sobre a atuação do biomédico, mude futuramente.

Em vista disso, outro artigo analisa o conhecimento dos usuários do Núcleo de Apoio à Saúde da Família que tiveram contato com biomédico e profissionais da saúde quanto a atuação do biomédico. De acordo com a pesquisa, os profissionais da saúde sabiam mais exemplos de atuação na atenção básica do que os usuários. Ademais, indicam que há uma falta de conhecimento do próprio profissional biomédico moldada pela formação acadêmica. Também, é sugerido a necessidade de o profissional conhecer sua função na atenção básica e dialogar com os outros profissionais. Além disso, é ressaltada a 
importância do biomédico nas orientações para coleta adequada de exames, interpretação de resultados e nas orientações de medidas de prevenção de doenças tanto durante os atendimentos quanto na atuação em grupos ou em escolas (Programa Saúde na Escola) por meio da educação em saúde, sendo eficaz o diálogo estabelecido com os usuários do SUS e com a população (Silva \& Júnior, 2019).

No que tange a dinâmica proposta para o Programa de Saúde da Família o cuidado se torna mais focado no usuário e, por isso, é mais eficiente para a promoção e recuperação da saúde e prevenção de agravos dentro de uma comunidade. (Costa et al., 2010). Ainda que as DCN da biomedicina proponham uma formação capaz de dar competências ao profissional para o atendimento no SUS, Silva e Júnior (2019) indicam que há necessidade de mudança nas diretrizes por haver maior prioridade por conhecimentos técnicos durante a graduação e que a inserção do biomédico na atenção básica é nova e, por isso, há necessidade que o curso e a categoria lutem por esse espaço como exemplo, pela inclusão em editais, conhecimento da equipe sobre a atuação na promoção da saúde e integração dos estudantes da área da saúde.

Silva et al. (2014) citando Barreto (2004) discorrem sobre a importância do biomédico dentro da saúde pública e indica decorrências do avanço das tecnologias em saúde, sugerindo reflexões para os profissionais:

"É evidente que nas últimas décadas, o sistema de saúde sofreu uma renovada dose de confiança, em paralelo ao avanço dos conhecimentos biomédicos aberto pelas novas possibilidades de estudo das doenças no nível molecular e genético. Contudo, esse sistema ainda traz questões como: (a) o seu crescente distanciamento da prevenção primária, pois, quanto mais se capacita para atuar sobre os efeitos da doença, mais se desaprende de como atuar sobre os seus determinantes; (b) os seus efeitos iatrogênicos, dos quais se reforçam evidências, (c) por fim, os seus custos crescentes, que não somente tornam as perspectivas macroeconômicas desse sistema sombrias, como também são bases das iniquidades de acesso, mesmo nas economias desenvolvidas (Barreto, 2004, p.2).”

Já sobre a educação em saúde, um estudo (Sousa \& Oliveira, 2017) traz essa discussão no campo da educação permanente através da análise do conhecimento de professores universitários sobre esse tema. Além disso, é introduzida a questão de os componentes curriculares da educação em saúde na grade dos cursos de biomedicina terem uma menor oferta que outras disciplinas de ciências biomédicas transparecendo uma ideia de menor importância. Para tal análise, o artigo construiu a discussão a partir do currículo das 15 disciplinas encontradas com relação direta a educação em saúde (somatórios de três Instituições de Ensino da Paraíba), observando-se diferenças quantitativas de disciplinas, mas com similaridade nas ementas; em seguida, foi exposta o perfil biodemográfico dos participantes do estudo. A partir disso, foi avaliado o conhecimento dos professores dessas disciplinas por meio da conceituação sobre educação em saúde exposta por eles. Como resultado, foi apresentada respostas limitadas e até mesmo a ausência de resposta para a questão sobre atividades desenvolvidas na Educação em Saúde. Por meio desse estudo, é possível observar que pode haver falhas no ensino de educação em saúde para os estudantes de Biomedicina que, consequentemente, impacta no exercício da profissão, já que, como visto através dos artigos incluídos neste estudo, a educação em saúde é uma das principais atuações do biomédico na promoção da saúde da população.

Carvalho et al. (2020) abordam uma forma de aplicação da prática da promoção da saúde durante a graduação. O artigo relata uma ação comunitária realizada por alunos ao cursar de uma disciplina em que é feito uma intervenção em uma escola da rede pública com a finalidade de promover a prevenção de enteroparasitoses. Os alunos desenvolvem a prática tanto laboratorial, por meio das análises das amostras, como em educação em saúde, proporcionando orientações sobre medidas profiláticas. No mais, essa prática estimula diversas competências já que os alunos são responsáveis pelas atividades desde a escolha da estratégia de intervenção até a análise dos resultados.

Esse estudo citado anteriormente dialoga com os outros artigos que abordam a extensão como pilar essencial para a formação acadêmica e, além disso, Martins et al. (2004) também discutem sobre o desenvolvimento da cidadania nos alunos 
por meio do exercício da educação em saúde que é fundamental para a formação dos estudantes e o retorno para a sociedade e traz relatos de respostas positivas das atividades.

Através das publicações incluídas nesse estudo, foi possível associar que o distanciamento da teoria com a prática é um dos maiores impeditivos para a atuação integral do profissional biomédico na promoção da saúde. Ademais os aspectos convergentes nas problemáticas apresentadas, o resultado de quatorze publicações corresponde ao possível desconhecimento sobre as funções do biomédico mesmo que as Diretrizes Curriculares Nacionais do curso tenham como destaque: “(...) formação generalista, humanista, crítica e reflexiva, para atuar em todos os níveis de atenção à saúde, com base no rigor científico e intelectual"

Esta revisão de escopo possui algumas limitações como as poucas publicações encontradas que relacionam os temas "promoção da saúde" e "biomedicina", sendo, os resultados tendo sido apenas encontrados no Google Acadêmico. O aumento dos termos de busca, possivelmente, possibilitaria um número de artigos incluídos maior, porém seria descartado uma grande quantidade de publicações que não possuem relação com o tema específico deste estudo. A abrangência da plataforma citada possibilitou a realização do estudo, uma vez que nas outras plataformas utilizadas não houve identificação de artigos, mesmo com a realização de um teste anterior com o uso de mais palavras-chave.

É possível também que esta revisão apresente aspectos de subjetividade ao definir e agrupar os assuntos discutidos nas diferentes publicações. Porém, a ação planejada e explícita na metodologia pode ter ajudado a diminuir a subjetividade inerente a qualquer tipo de estudo.

\section{Conclusão}

A presente revisão de escopo sistematizou as evidências conhecidas e identificar as lacunas presentes na relação profissional biomédico e promoção da saúde. Mesmo que uma parcela dos artigos tenha foco na atuação do biomédico, como identificado na análise, todos indicam fragilidades e efeitos oriundos da formação acadêmica. Desse modo, foi indicado que uma maior integração entre os cursos da área da saúde, vivência prática com a aplicação para a sociedade, estudos com perspectiva antropológica e ênfase na interdisciplinaridade auxiliariam em uma formação mais ampliada para o exercício do profissional biomédico na promoção da saúde.

É imprescindível que os estudantes de biomedicina tenham conhecimento de lacunas da sua formação para que busque preenchê-las. O pilar das universidades já é muito difundido como ensino, pesquisa e extensão, mas a vivência ao longo da graduação indica que é necessário fazer-se presente para além das disciplinas e atividades ofertadas pelo curso. O pensamento crítico e o cuidado humanizado devem ser estimulados pelo curso, embora seu exercício também dependa do aluno.

Sugere-se que sejam realizados mais estudos empíricos abordando a relação profissional biomédico e promoção da saúde, uma vez que esta revisão identificou poucos trabalhos com foco no citado tema. Por fim, deve ser destacado que as posições expressas nesse artigo pertencem exclusivamente às autoras e não refletem, necessariamente, as visões da instituição às quais estão vinculadas.

\section{Referências}

Almeida Filho, N. (2011). O que é saúde? Editora Fiocruz.

Backes, D. S., Carpes, A. D., Piovesan, C., Haeffner, L. S. B., Büscher, A., \& Lomba, L. (2014). Trabalho em equipe multiprofissional na saúde: da concepção ao desafio do fazer na prática. Disciplinarum Scientia Saúde, 15(2), 277-289.

Bezerra, I. M. P., \& Sorpreso, I. C. E. (2016). Conceitos de saúde e movimentos de promoção da saúde em busca da reorientação de práticas. Journal of Human Growth and Development, 26(1), 11-20. 
Brasil. Lei $n^{0}$ 8080, 19 de setembro de 1990. (1990). Brasília.

Brasil. Ministério da Saúde. Conselho Nacional de Saúde. Resolução CNS nº 286, de 8 de outubro de 1998. (1998). Brasília.

Brasil. Ministério da Saúde. Política Nacional de Promoção da Saúde / Ministério da Saúde, Secretaria de Vigilância em Saúde, Secretaria de Atenção à Saúde. (3a ed.), Ministério da Saúde, 2010.

Brasil. Ministério da Saúde. Secretaria de Vigilância em Saúde. Secretaria de Atenção à Saúde. Câmara Nacional de Educação. Conselho Nacional de Educação. Resolução CNE/CES n n 2, de 18 de fevereiro de 2003. Brasília.

Buss, P. M. Promoção da saúde e qualidade de vida. (2000). Ciência \& Saúde Coletiva, 5 (1), 163-177.

Cardozo, M. Q., Gomes, K. M., Fan, L. G., \& Soratto, M.T. (2016). Fatores associados à ocorrência de ansiedade dos acadêmicos de Biomedicina. Saúde e Pesquisa, 9(2): 251-262.

Carvalho, S. R. Os múltiplos sentidos da categoria" empowerment" no projeto de Promoção à Saúde. (2004). Cadernos de Saúde Pública, v. 20(4), 1088-1095

Carvalho, W. V., Martins, Y. S., Silva, P. L. D., Cristovam, F. A., \& Carvalho, A. F. Atenção às ações comunitárias na formação de biomédicos. (2020). Revista Brasileira de Ciências Biomédicas, 1(1), 1-6.

Chiesa, A. M., Nascimento, D. D. G., Braccialli, L. A. D., Oliveira, M. A. C., \& Ciampone, M. H. T. (2007). A formação de profissionais da saúde: aprendizagem significativa à luz da promoção da saúde. Cogitare enfermagem, 12(2), 236-240.

Costa, F. B; Trindade, M. A. N., \& Pereira, M. L. T. (2010). A inserção do biomédico no programa de saúde da família. Revista Eletrônica Novo Enfoque, 11 (11), 27-33.

Czeresnia, D. (2003). O conceito de saúde e a diferença entre prevenção e promoção. In Czeresnia, D.\& Freitas, C. M. (Orgs.). Promoção da saúde: conceitos, reflexões, tendências (pp. 39-53). Fiocruz.

Faria, A. M. B., Valiatti, T. B., Oliveira, A. A., \& Salvi, J. O. (2017). A fitoterapia entre acadêmicos das ciências da vida. Revista Saúde e Desenvolvimento 11(9), 198-213.

Heidmann, I. T. S. B., Almeida, M. C. P., Boehs, A. E., Wosny, A. M., \& Monticelli, M. (2006). Promoção à saúde: trajetória histórica de suas concepções. Texto \& Contexto-Enfermagem, 15(2), 352-358.

Larson, K.; Jung, S. G., \& Albon, S. (2019). Searching the literature: a simple step-wise process for evidence-based medicine. Journal of Pharmacy Technology, 35(5), 225-229.

Martins, C. H. G., Cano, M. A. T., Moura, S. M. B. L., Guagneli, Z. M. S., \& Demacq, S. (2004). Projeto Cidadania: formação do profissional biomédico. Revista Em Extensão, 4(1), 91-95.

Munn, Z., Peters, M. D. J., Stern, C. Tufanaru, C., McArthur, A. \& Aromataris, E. (2018). Systematic review or scoping review? Guidance for authors when choosing between a systematic or scoping review approach. BMC Medical Research Methodology, 18(143), https://doi.org/10.1186/s12874-018-0611-x.

Oliveira, M. M. S., Simões, A. L. A., Contim, D., Goulart, B. F., \& Ruiz, M. T. (2021). Liga acadêmica de humanização e a formação profissional em saúde: percepções de ligantes egressos. Research, Society and Development, 10 (11), e481101120003.

Perinazzo, J., Sandri, Y. P., Mallet, E. K. V., \& Zimmerman, C. E. P. (2015). A atuação do profissional biomédico na atenção primária à saúde: desafios na formação. Revista Saúde Integrada, 8 (15-16).

Pessoa, W. D., Mota, J. C., \& Samá, S. (2018). A integração da Educação Ambiental na formação do biomédico em uma Universidade comunitária: indícios a partir dos Princípios da Ambientalização Curricular. REMEA-Revista Eletrônica do Mestrado em Educação Ambiental, 1, 4-19.

Pinheiro, D. G. M., Scabar, T. G., Maeda, S. T., Fracolli, L. A., Pelicioni, M. C. F., \& Chiesa, A. M. (2015). Competências em promoção da saúde: desafios da formação. Saúde e Sociedade, v. 24(1), 180-188.

Rodrigues, G. P., Durigon, G. S., Lisboa, M. L., Bodanezi, A., Barros, B. A. C. Duque, T. M., Munhoz, E. A., Rath, I. B. S., \& Camargo, A. R. (2018). Impacto de um projeto de extensão sobre a formação discente para atuação em ambiente hospitalar. Extensio: Revista Eletrônica de Extensão, 15(31), 67-78.

Santos, C. O. \& Melo, A. T. (2020). A importância do profissional biomédico na prática de Cuidados Paliativos no tratamento oncológico. (2020). Brazilian Journal of Development, 6(11), 92651-92664.

Silva, A. R., Nunes, C. R. S., Araújo, S. S., \& Veras, H. N. H. (2014). O papel do biomédico na Saúde Pública. Revista Interfaces: Saúde, Humanas e Tecnologia, 2(4), 1-5.

Silva, C. A., \& Júnior, D. D. L. F. (2019). Inserção do biomédico nas equipes multiprofissionais da Estratégia de Saúde da Família e Núcleo de Apoio à Saúde da Família. Revista Saúde Integrada, 12(23), 90-100.

Silva, C. J. A., Ventura, A. F., \& Costa Júnior, C. E. O. (2015). O papel do Biomédico na análise ambiental. Caderno de Graduação-Ciências Biológicas e da Saúde-UNIT-Pernambuco, 2(1), 11-20.

Silva, K. W. L., Silva, S. K. O., Gomes, D. C. S., Silva, K. K. L., Santos, A. J. S., Silva, D. C., Barbosa, J. I. S., Lopes, V. C. M., Costa, J. R. M., \& Fonseca, S. A. (2019). Liga acadêmica de plantas medicinais: um desafio extracurricular. Extensio: Revista Eletrônica de Extensão, 16(34), 85-102.

Sousa, A. P. \& Oliveira, S. F. (2017). Avaliação do conhecimento de professores universitários de cursos de biomedicina sobre educação em saúde. Temas em Saúde, 17(4), 96-112. 
Research, Society and Development, v. 11, n. 3, e44811326598, 2022

(CC BY 4.0) | ISSN 2525-3409 | DOI: http://dx.doi.org/10.33448/rsd-v11i3.26598

Souza, J. M., Tholl, A. D., Córdova, F. P., Heidemann, I. T. S. B., Boehs, A, E., \& Nitschke, R. G. (2014). Aplicabilidade prática do empowerment nas estratégias de promoção da saúde. Ciência \& saúde coletiva, 19(7), 2265-2276.

Tesser, C. D. Práticas complementares, racionalidades médicas e promoção da saúde: contribuições poucos exploradas. (2009). Cadernos de Saúde Pública, 25(8), 1732-1742.

Tricco, A.C., Lillie, E., Zarin, W., O'Brien, K.K., Colquhoun, H., Levac, D., Moher, D., Peters, M. D., Horsley, T., Weeks, L., Hempel, S., Akl, E. A. et al. (2018). PRISMA extension for scoping reviews (PRISMA-ScR): checklist and explanation. Annals of Internal Medicine, 169(7), 467-473. 